
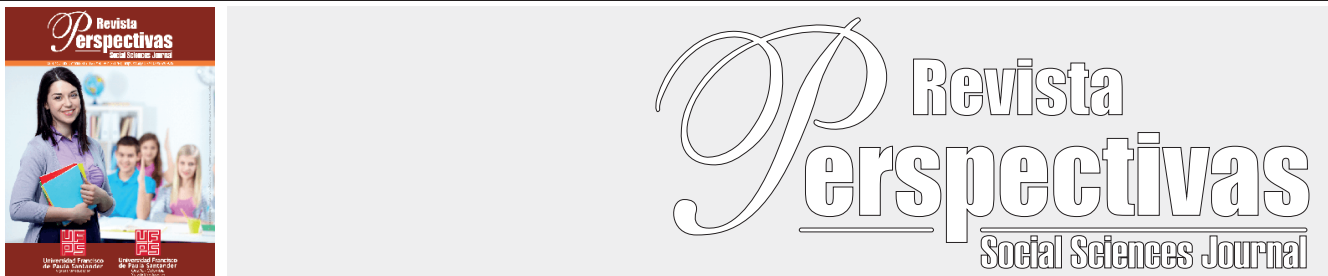

Artículo Original

https://doi.org/10.22463/0122820X.1793

\title{
Juego y enseñanza de las Matemáticas: Reflexiones teóricas para el trabajo de aula
}

Game and teaching of mathematics: theoretical reflections for classroom work

Angélica María Peñaranda Ramírez', Raúl Prada Núñez², Audin Aloiso Gamboa Suárez ${ }^{3}$

${ }^{1}$ Licenciada en Pedagogía Infantil, 94anmapera@gmail.com, ORCID: 0000-0001-5739-8636, Universidad de los Llanos, Villavicencio, Colombia.

${ }^{2}$ Magister en Educación Matemática, raulprada@ufps.edu.co, ORCID: 0000-0001-6145-1786, Universidad Francisco de Paula Santander, Cúcuta, Colombia.

${ }^{3}$ Doctor en Ciencias de la Educación, audingamboa@ufps.edu.co, ORCID: 0000-0001-9755-6408, Universidad Francisco de Paula Santander, Cúcuta, Colombia.

Cómo citar: A.M Peñaranda Ramírez, R. Prada Núñez, A.A Gamboa Suárez, "Juego y enseñanza de las Matemáticas: Reflexiones teóricas para el trabajo de aula". Perspectivas, vol. 4, no. 2, pp. 80-84, 2019.

Recibido: Junio 30, 2018; Aprobado: Octubre 11, 2018.

\begin{tabular}{ll}
\hline & RESUMEN \\
\hline Palabras clave: & El presente artículo hace parte de una amplia investigación titulada juegos tradicionales como estrategia de \\
evaluación en el aprendizaje de las matemáticas en educación básica primaria. Se muestra una reflexión sobre \\
enseñanza; innovación; & las diferentes posibilidades metodológicas que se pueden utilizar en el aula para la enseñanza de las matemáticas \\
educación matemática; & desde una postura lúdico-didáctica que pretende conferir al maestro diferentes medios de enseñanza de esta \\
juegos tradicionales. & asignatura del currículo escolar. La revisión teórica se realiza a partir de autores relevantes en el campo como \\
& Subiza, Piaget y Espinoza. Se concluye que el juego tradicional como estrategia en la enseñanza es un elemento \\
& innovador en los contextos escolares y puede considerarse de mejor aceptación en el momento de evaluar los \\
& aprendizajes de los estudiantes.
\end{tabular}

\begin{tabular}{ll}
\hline ABSTRACT \\
\hline Keywords: & $\begin{array}{l}\text { This article is part of an extensive investigation titled traditional games as an evaluation strategy in learning } \\
\text { mathematics in primary education. A reflection is shown on the different methodological possibilities that can } \\
\text { be used in the classroom for teaching mathematics from a playful-didactic position that aims to confer on the }\end{array}$ \\
teaching; innovation; & $\begin{array}{l}\text { teacher different means of teaching this subject in the school curriculum. The theoretical review is carried out } \\
\text { from relevant authors in the field such as Subiza, Piaget and Espinoza. It is concluded that traditional play as } \\
\text { Mathematics education; } \\
\text { traditional games. }\end{array}$ \\
& $\begin{array}{l}\text { a teaching strategy is an innovative element in school contexts and can be considered as better accepted when } \\
\text { evaluating student learning. }\end{array}$
\end{tabular}

\section{Introducción}

En Latinoamérica es común ver a los niños en las calles, barrios y patios de escuela divirtiéndose con una diversidad importante de juegos tradicionales que se ven en cualquier país, en función a la cultura desarrollada en cada uno de estos, si bien con alguna u otra diferencia como en su nombre o diseño, pero al final la misma esencia.

Según Pérez (1997), en las barriadas y patios de las escuelas se genera un caudal importante de juegos populares, lo que constituye un real tesoro infantil y juvenil. En realidad, es tal la producción lúdica que se configura a partir de la práctica asociada a las formas de la imaginación, que muchos de estos juegos alentados de esa manera, llega a transmitirse de una generación a otra.

En la mayoría de las escuelas como en los hogares en Latinoamérica los juegos tradicionales están presentes en su cotidianidad, por lo que es aplicado como actividades recreativas en las horas de descanso o medio motivador en el aula de clase, porque el juego es parte de la naturaleza del hombre y una forma de aprendizaje. Calero (2003), afirma que en muchas de las escuelas se prepondera el valor del aprendizaje pasivo, domesticador y alienante; no 
se da la importancia del caso a la educación integral y permanente.

La escuela tradicionalista asume que la enseñanza en los niños debe ser aplicada por parte de los profesores con rigidez, obediencia ciega, criticidad, pasividad y ausencia de iniciativa. Por el logocentrismo, algunas veces el juego está vedado, en el mejor de los casos, admitido solamente en el horario del recreo. Por tanto, los juegos son una estrategia útil en los procesos de aprendizaje en el aula de clase, porque estos permiten adaptarse a cualquier contenido despertando la atención de los estudiantes y perimiendo la comprensión de conceptos y el desarrollo de competencias.

Aunque en general los juegos tradicionales son adaptables en los procesos de aprendizaje se debe tener en cuenta el contexto de su aplicación, la edad de los niños, el espacio y material a utilizar de acuerdo a lo que se va a realizar. Una de las áreas a las que más se adapta el juego es en el área de las Matemáticas y Ciencias Exactas ya que si se hace un análisis de las características en el desarrollo de estos campos se puede destacar el aprendizaje de relaciones numéricas, las relaciones geométricas y la solución de problemas (Hernández-Suárez, PradaNúñez \& Gamboa-Suárez, 2017; Vergel-Ortega, Hernández \& Rincón-Leal, 2016).

De acuerdo a estudios de la Organización para la Cooperación y el Desarrollo Económicos (OCDE, 2016) los 64 países participantes en el Programa para la Evaluación Internacional de los Alumnos (PISA, por sus siglas en inglés), señala que Latinoamérica está por debajo de los estándares globales de rendimiento escolar en áreas como Ciencias, Matemáticas y Lenguaje, en los cuales Perú, Colombia, Brasil y Argentina se encuentran entre los diez países cuyos estudiantes tienen un nivel bajo en áreas como las matemáticas, la ciencia y la lectura. De acuerdo al informe de la (OCDE, 2016) deduce que estos bajos puntajes en las escuelas en Latinoamérica, hacen parte de la falta de formación de los estudiantes, pero también de los maestros (Gamboa Suárez, Hernández Suárez \& Prada Núñez, 2018; Cifuentes Robledo y Villa Ochoa, 2018; Montes Miranda, Ramos González y Casarrubia Ruiz, 2018; Gamboa-Suárez, 2016).

De acuerdo con estos argumentos, los problemas del contexto y la necesidad que muestran los estudiantes en la compresión de las matemáticas se proponen una estrategia lúdica - académica para que el docente se apropie de herramientas como los juegos tradicionales para fortalecer la enseñanza de las matemáticas (García-Quintero \& Villamizar Suárez, 2017).

\section{Aproximación al concepto de juego}

Espinoza y Espinoza (2005), señalan que el juego es una acción u ocupación libre, que se desarrolla dentro de unos límites temporales y espaciales determinados, según reglas absolutamente obligatorias, aunque libremente aceptadas, acción que tiene su fin en sí misma y va acompañada de un sentimiento de tensión y alegría y de la conciencia de ser de otro modo en la vida cotidiana.

El concepto de juego ha sido aplicado en distintas situaciones para explicar funciones de recreación, de desarrollo motor y hasta el nivel cognoscitivo de una persona, este término no es nuevo, ya que a través del tiempo y de acuerdo a cada autor representa una acción importante en la vida del ser humano desde el desarrollo de sus funciones para la vida adulta como su desarrollo cognoscitivo de acuerdo a su desarrollo multidimensional y como una actividad u ocupación libre y espontanea.

En cuanto a las características del juego, Díaz (2002) afirma que el juego es un espacio liberador, no tiene interés material, se desarrolla con orden, el juego manifiesta regularidad y consistencia, tiene límites que la propia trama estable y se auto promueve. Del mismo modo, el autor manifiesta que otras de las características del juego se manifiestan como 
una fantasía hecha realidad, es una reproducción de la realidad en el plano de la ficción. Se expresa en un tiempo y un espacio, el juego no es una ficción absoluta, puede ser individual o social, es evolutivo y por su puesto es una forma de comunicación.

Por su parte Caillois (1996), señala que el juego es una actividad que posee reglas pero que se caracteriza por ser libre, nadie puede obligarte a jugar, es una decisión voluntaria que separa la vida cotidiana en un espacio y tiempo específicos, es incierta ya que no está determinada, improductiva pues no crea bienes ni riquezas fuera del juego, cuando se termina, las cosas vuelven a empezar como la primera vez.

\section{Juego y Cognición}

Piaget (1956), relaciona el juego con su teoría cognoscitiva, mostrándolo como una actividad que favorece el desarrollo del conocimiento asociándolo de tal forma a sus

tres estructuras básicas con las fases evolutivas del pensamiento humano: el juego es simple ejercicio (parecido al anima); el juego simbólico (abstracto, ficticio); y el juego es reglado (colectivo, resultado de un acuerdo de grupo).

Cada etapa supone la consistencia y la armonía de todas las funciones cognitivas en relación a un determinado nivel de desarrollo. También implica discontinuidad, hecho que supone que cada etapa sucesiva es cualitativamente diferente al anterior, incluso teniendo en cuenta que, durante la transición de una etapa a otra, se pueden construir e incorporar elementos de la etapa anterior. Piaget (1956), divide el desarrollo cognitivo en cuatro etapas: la etapa sensomotriz (desde el nacimiento hasta los dos años), la etapa pre operativa (de los dos a los seis años), la etapa operativa o concreta (de los seis o siete años hasta los once) y la etapa del pensamiento operativo formal (desde los doce años aproximadamente en lo sucesivo).
Piaget (1961), argumenta que los juegos de reglas van a integrar y combinar todas las destrezas adquiridas: combinaciones sensorio-motoras (carreras, lanzamientos, entre otras) o intelectuales (ajedrez) con el añadido de la competitividad ( $\sin$ la que la regla no sería de utilidad) y bajo la regularización de un código normativo vinculado a la naturaleza del propio juego o por simples pactos puntuales e improvisados. La regla que sostiene Piaget, resulta de la organización colectiva de las actividades lúdicas.

Subiza (1991), por su parte muestra una perspectiva diferente a los tipos de juegos, los cuales se ven en la cotidianidad como los juegos tradicionales que pasan de generaciones y se vuelven populares, pero no solo pasan de ser una actividad de casa o tiempo libre. Piaget (1956), los relaciona con su teoría del desarrollo multidimensional para dar a entender el tipo de juego que se puede llegar aplicar de acuerdo a cada persona. En esta propuesta Piaget aporta no solo a la teoría del desarrollo multidimensional para conocer de acuerdo a cada edad que tipo de juego se puede aplicar, sino también las habilidades que desarrolla el juego reglado, dando así la posibilidad de aplicar los tipos de juegos propuesto por Subiza (1991) como una alternativa innovadora en la educación.

\section{Juego y Enseñanza de las Matemáticas}

En este aparte, se reafirma la importancia del juego en la enseñanza de las ciencias y concretamente en la matemática. En este sentido importantes estudios como los realizados por López (2018) concluye que

El docente debe asumir la enseñanza de la matemática desde estrategias didácticas lúdicas, que impacten, motiven y le hagan más accesible el conocimiento al estudiante, que le aseguren la comprensión de los conceptos y principios 
matemáticos para su aplicación en la vida cotidiana (p.68).

De otro modo, Morales y Villa (2019) proponen una herramienta muy interesante y la denominan juego de roles para la enseñanza de las matemáticas, esta herramienta emplea actividades lúdicas basadas en el sistema de juegos de roles y hace atractivo el proceso de aprendizaje. Se fomenta el trabajo colaborativo donde los integrantes resuelven situaciones ficticias, establecidas por el profesor, donde se abordan temáticas de las áreas de las Ciencias y del área de Matemáticas.

Un aspecto más concreto en el marco de enseñanza de las Matemáticas es el desarrollo de los distintos pensamientos matemáticos (HernándezSuárez, Prada-Núñez \& Ramírez-Leal, 2017). En este sentido, Aristizábal, Colorato y Gutiérrez (2016), demuestran que, con base en nueva estrategia metodológica como el juego, las matemáticas adquieren un nuevo sentido para el estudiante. Los autores hacen una recomendación importante como resultado de esta investigación y señalan que

La enseñanza de las matemáticas en estos momentos de cambio, deben reflexionar sobre los métodos didácticos convencionales utilizados en el aula de clase, y procurar por la transformación del proceso de enseñanza-aprendizaje y la forma en que docentes y alumnos acceden al conocimiento ( $\mathrm{p}$. 136).

Es importante señalar que es amplio el abanico de estudios y teorías donde se muestran las bondades del juego en la enseñanza de las matemáticas con estrategias novedosas como el crucigrama (Medina y Delgado, 2020), los jugos mentales (Rodríguez, 2020) y los juegos tradicionales (Chilclón, 2020)

\section{Conclusiones}

El juego tradicional como estrategia en la enseñanza de las matemáticas puede ser considerado innovador para muchos docentes, pero muy pocos toman realmente su importancia y el tiempo para ejecutarlo como una estrategia que no sea simplemente de reacción, sino como un elemento para enseñar.

La creatividad tiene gran relevancia en la educación puesto que no tiene límite cuando se enseña, el juego potencializa la creatividad y de esta manera los estudiantes pueden aprender de forma significativa y su visión de alta complejidad sobre las matemáticas puede cambiar desde una mirada lúdica, sin dejar, por supuesto, el rigor que exige el área.

Del mismo modo, es importante señalar que la lúdica en la enseñanza de las diferentes áreas proporcionan luces importantes en temas de formación de maestros desde la utilización de manuales para juegos tradicionales como estrategia de aprendizaje en el área de matemáticas en básica primaria.

\section{Referencias}

Aristizábal, J., Colorato, H. \& Gutiérrez, H. (2016). El juego como una estrategia didáctica para desarrollar el pensamiento numérico en las cuatro operaciones básicas. Sophia, 12(1), 117-125. DOI: https://doi.org/10.18634/ sophiaj.12v.1i.450

Calero, M. (2003). Juego de crianza: el juego corporal en los primeros años de vida. Buenos Aires, Argentina: Editorial Biblios.

Caillois, R (1996). Teoría de los Juegos. Barcelona, España: Editorial Seis Barral.

Chilcon Flores, F. (2020). Juegos tradicionales para desarrollar el aprendizaje de la matemática en los niños de 5 años, de la institución educativa inicial No 951 "Niño Dios" de la provincia de Cutervo, año 2018. (Tesis de pregrado). ULADECH, Chile

Cifuentes-Robledo, W. y Villa-Ochoa, J. (2018). Características de los modos de pensamiento matemático en profesores de matemáticas. Revista Espacios, 39(10), 6-23. Recuperado: 
https://www.revistaespacios.com/a18v39n10/ a18v39n10p06.pdf

Díaz, F. A. (2002). Estrategias docentes para un aprendizaje significativo. México: McGraw-Hill.

Espinoza, M. R., \& Espinoza, M. C. R. (2009). Johan Huizinga (1872-1945): Ideal caballeresco, juego y cultura. Konvergencias: Revista de Filosofía y Culturas en Diálogo, (21), 3-18. Recuperado de https://dialnet.unirioja.es/servlet/ articulo? codigo $=3053746$

Gamboa-Suárez, A. A. (2016). Docencia, investigación y gestión: Reflexiones sobre su papel en la calidad de la educación superior. Revista Perspectivas, 1(1), 81-90. https://doi. org/10.22463/25909215.973

Gamboa Suárez, A., Hernández Suárez, C., \& Prada Núñez, R. (2018). Práctica pedagógica y competencias TIC. Saber, Ciencia Y Libertad, 13(1), 258-274. https://doi.org/10.18041/23823240/saber.2018v13n1.2090

García-Quintero, C., \& Villamizar Suárez, G. (2017). Análisis fenomenológico de la conciencia del docente a partir de sus prácticas evaluativas. Revista Perspectivas, 2(2), 49-59. https://doi. org/10.22463/25909215.1313

Hernández-Suárez, C. A., Prada-Núñez, R., \& Gamboa-Suárez, A. A. (2017). Knowledge and use of mathematical language in initial teacher training in mathematics. Revista de Investigación, Desarrollo e Innovación, 7(2).

Hernández-Suárez, C. A., Prada-Núñez, R., \& Ramírez-Leal, P. (2017). Obstáculos epistemológicos sobre los conceptos de límite y continuidad en cursos de cálculo diferencial en programas de ingeniería. Revista Perspectivas, 2(2), 73-83. https://doi. org/10.22463/25909215.1316

López Montero, M. (2018). 4. El juego como estrategia didáctica para la enseñanza de la matemática en educación especial. Revista EDUCARE - UPEL-IPB - Segunda Nueva Etapa 2.0, 21(2), 70-90. DOI: https://doi.org/10.46498/ reduipb.v21i2.65

Medina-Cepeda, N. M., \& Delgado, J. R. (2020). El
Crucigrama como estrategia para la enseñanza y aprendizaje de la matemática universitaria. CienciAmérica, 9(1), 11-33. Recuperado de http://201.159.222.118/openjournal/index.php/ uti/article/download/243/381 ?inline $=1$

Montes Miranda, A.J., Ramos González, D.M. y Casarrubia Ruíz, J.L. (2018). La formación de maestros en Colombia: alcances y limitaciones. Revista Espacios, 39(10), 6-23. Recuperado: https://www.revistaespacios.com/ a18v39n10/18391021.html

Morales, R. \& Villa, C. (2019). Juegos de rol para la enseñanza de las matemáticas. Education in the knowledge society (EKS), (20), 7. DOI: https:// doi.org/10.14201/eks2019_20_a7

OCDE. (2016). La educación en Colombia. Recuperado de: www. mineducacion.gov.co articulo 356787.

Piaget, J. (1956). Etapa de Piaget y los juegos. Recuperado de: https://emowe.com/juegosetapas- de-piaget/.

Piaget, J. (1961). El juego infantil y su metodología. Recuperado de: https//books.google.com.co/ Brooks.

Pérez, A. (1997). Recreación y fundamentos teóricos metodológicos. México: Instituto Politécnico Nacional.

Rodríguez, D. (2020). El impacto de los juegos mentales para el aprendizaje de las matemáticas. Recuperado de https://www.normaldesaboya. edu.co/images/publicaciones/pintando escuelas_version_final.pdf\#page $=55$

Subiza, J.M. (1991). Juegos tradicionales en el Valle de Estereibar a Ortazadar. Recuperado de: http:// www.efdeportes.com/efd179/propuestas-declasificacion-del-juego.htm.

Vergel-Ortega, M., Hernández, R. V., \& RincónLeal, O. L. (2016). Influencia de curso precálculo y actividades de apoyo institucional en desarrollo de competencias y creencias en matemáticas. ECOMATEMATICO, 7(1), 33-47. https://doi. org/10.22463/17948231.1099 